\title{
Cell Wash
}

National Cancer Institute

\section{Source}

National Cancer Institute. Cell Wash. NCI Thesaurus. Code C76322.

The process of applying a solvent (e.g., water) or a solution (e.g., SSC/SDS) to a biomaterial or an array to remove impurities or unwanted compounds. 